\title{
Study of desmoglein 1 and 3 antibody levels in relation to disease severity in Indian patients with pemphigus
}

\author{
Bhushan Kumar, Sunil Arora*, Muthu Sendhil Kumaran, Rajesh Jain, Sunil Dogra \\ Departments of Dermatology, Venereology and Leprology and *Immunopathology, Postgraduate Institute of Medical Education \\ and Research, Chandigarh, India.
}

Address for correspondence: Prof. Bhushan Kumar, Former Head, Department of Dermatology, Venereology and Leprology, PGIMER, H. No. 81, Sector 16, Chandigarh 160 015, India. E-mail: kumarbhushan@hotmail.com

\section{ABSTRACT}

Objectives: To conduct a cross-sectional study to compare Dsg1 and Dsg3 antibody levels independently with severity of disease activity in pemphigus vulgaris (PV) and pemphigus foliaceus (PF). Methods: Blood samples from 44 patients with pemphigus (PV-38, PF-6) were analyzed using ELISA. The severity of skin and mucosal disease was graded using a score from 0 to 3. Results: A statistically significant correlation between increase in Dsg 3 antibody titres with severity of oral involvement and Dsg 1 titres with severity of skin involvement was found in both PV and PF patients $(p<0.01)$. However, we were unable to demonstrate a relationship between increased titres of Dsg1 and Dsg 3 antibodies with oral and skin involvement respectively. Conclusion: This study suggests that the severity of skin and oral disease in pemphigus is determined by the quantities of Dsg1 and Dsg3 antibodies respectively.

Key Words: Antibodies, Desmoglein 1 and 3, Pemphigus, Serology

\section{INTRODUCTION}

Pemphigus is one of the best-characterized tissuespecific autoimmune diseases. The desmoglein proteins desmoglein-3 (Dsg3) and desmoglein-1 (Dsg1) have been identified as the antigens in pemphigus vulgaris (PV) and pemphigus foliaceus (PF) respectively. While earlier studies suggested that indirect immunofluorescence titers were a useful marker for disease activity in pemphigus, ${ }^{[1-3]}$ a detailed study concluded that serial titers were not consistent enough to be used as a guide for therapy and prognosis. ${ }^{[4]}$ The production of recombinant Dsg 1 and Dsg3 molecules has provided the opportunity to determine levels of antibodies to them (anti-Dsg1 IgG and anti-Dsg3 $\operatorname{IgG}$ respectively) and to see if they correlate with disease severity. ${ }^{[5]}$ However, there are conflicting data in this regard in many studies using immunofluorescence titers. ${ }^{[1-3]}$

ELISA is a simple and effective tool for the quantitative analysis of antibody levels. Harman et al. used ELISA to measure serial Dsg1 and Dsg3 antibody levels and correlate them with the severity of oral and skin ulceration in $\mathrm{PV}$ and $\mathrm{PF} \cdot{ }^{[6]}$ As there are hardly any similar studies from India, we conducted this cross-sectional study to correlate the severity of oral and/or cutaneous involvement in patients with PV and PF with ELISA

How to cite this article: Kumar B, Arora S, Kumaran MS, Jain R, Dogra S. Study of desmoglein 1 and 3 antibody levels in relation to disease severity in Indian patients with pemphigus. Indian J Dermatol Venereol Leprol 2006;72:203-6.

Received: October, 2005. Accepted: February, 2006. Source of Support: Nil. Conflict of interest: None declared. 
values for antibodies to Dsg1 and Dsg3 independently.

\section{METHODS}

Forty-four patients with pemphigus (38 with PV and 6 with PF, diagnosed based on clinical examination, histopathology and direct immunofluorescence) and three patients with extensive burns (as controls) were studied. We chose patients with burns as controls because pemphigus-like circulating intercellular antibodies have been found in such patients. ELISA was used to confirm the presence of Dsg1 and Dsg3 autoantibodies in PF and PV patients and an attempt was made to correlate their levels with cutaneous or mucosal involvement and disease activity. The blood samples were taken from patients who were untreated and with active disease or from those with resolving disease on treatment of varying durations. No serial samples were taken from any patient.

A simple arbitrary scoring system was used to grade the severity of skin or mucosal involvement at the time of sampling as follows:

\section{Oral score}

$0=$ No mucosal involvement.

1 = Minimal disease (only buccal mucosal, labiogingival, lingual, palatal or pharyngeal involvement).

2 = Moderate disease (buccal and labiogingival, lingual, palatal or pharyngeal involvement).

$3=$ Severe disease (extensive oral erosions, i.e., $>3$ mucosal sites affected)

\section{Skin score}

$0=$ Quiescent disease.

$1=$ Minimal disease $(\leq 10 \%$ body surface area involvement (BSA) involved).

$2=$ Moderate disease (11-30\% BSA involved).

$3=$ Severe disease: (>30\% BSA involved).

Antibodies to Dsg1 and Dsg3 were detected in the sera of patients by the micro ELISA technique using kits from Medical and Biological Laboratories Co. Ltd., Japan, as per the manufacturer's instructions. ODs were adjusted relative to the reference sera supplied with the kits.

Forty-seven serum samples were analyzed. The serum samples were randomly taken and subsequently stored at $-20^{\circ} \mathrm{C}$ before processing them for ELISA.

The unpaired t test was used to compare Dsg1 and Dsg3 levels in relation to severity of either skin or mucosal lesions.

\section{RESULTS}

A cut off value for each ELISA was established from the mean $\pm 2 \mathrm{SD}$ of the three patients with extensive burns. Although the cut off values given in the kit were taken as standard, to increase the sensitivity, we wanted to generate a cut off value from the local population as the kit was manufactured elsewhere. For Dsg1 ELISA, the mean was 0.143 and for Dsg3 it was 0.102 . This resulted in a cut off value of 0.406 for Dsg1 ELISA and 0.212 for Dsg3 ELISA. Dsg1 and Dsg3 antibodies were found to be present in all the patients of PV and PF. A summary of the disease and demographic parameters is given in Table 1.

For Dsg1 ELISA, 5 of 6 (83.3\%) PF patients and 23 of 38 (60.52\%) PV patients were above the cut off values. For Dsg3 ELISA, 31 of 38 (81.57\%) PV patients and 1 of 6 (16.6\%) PF patients exceeded the cut off value.

\section{ELISA values and disease severity}

We attempted to correlate the extent of skin and mucosal involvement [Table 2] with the independent values of Dsg1 and Dsg3 ELISA [Table 3]. Although a wide range of values was obtained, the general trend was that Dsg3 levels were higher in patients with extensive oral mucosal involvement, i.e., patients with moderate and severe mucosal involvement had

Table 1: Disease and demographic parameters

\begin{tabular}{lcc}
\hline & $\begin{array}{c}\text { Pemphigus vulgaris } \\
(\mathrm{n}=38)\end{array}$ & $\begin{array}{c}\text { Pemphigus foliaceus } \\
(\mathrm{n}=6)\end{array}$ \\
\hline Males & 18 & 4 \\
Females & 20 & 2 \\
Age (years) & $10-52$ & $15-50$ \\
Active disease (untreated) & 26 & 3 \\
Healing/inactive disease & 12 & 3 \\
\hline
\end{tabular}


Table 2: Oral disease severity and ELISA levels for antibodies to Dsg1 and Dsg3

\begin{tabular}{lcccc}
\hline $\begin{array}{l}\text { Oral mucosal } \\
\text { involvement }\end{array}$ & PV & PF & $\begin{array}{c}\text { Mean Dsg1 } \\
\text { antibody levels }\end{array}$ & $\begin{array}{c}\text { Mean Dsg3 } \\
\text { antibody levels }\end{array}$ \\
\hline None & 0 & 6 & 1.17 & 0.33 \\
Mild & 8 & 0 & 0.60 & 1.01 \\
Moderate & 11 & 0 & 0.77 & 1.27 \\
Severe & 19 & 0 & 1.25 & 2.58 \\
\hline
\end{tabular}

Dsg1: $p>0.1$; Dsg3: $p<0.01$; PV-Pemphigus vulgaris; PF-Pemphigus foliaceus

Table 3: Extent of skin involvement and ELISA levels for antibodies to Dsg1 and Dsg3

\begin{tabular}{lcccc}
\hline $\begin{array}{l}\text { Extent of skin } \\
\text { involvement (BSA) }\end{array}$ & PV & PF & $\begin{array}{c}\text { Mean Dsg1 } \\
\text { antibody levels }\end{array}$ & $\begin{array}{c}\text { Mean Dsg3 } \\
\text { antibody levels }\end{array}$ \\
\hline Mild & 14 & 1 & 0.31 & 1.04 \\
Moderate & 20 & 3 & 1.36 & 1.88 \\
Severe & 4 & 2 & 1.84 & 2.51 \\
\hline
\end{tabular}

Dsg1: $p<0.01$; Dsg3: $p>0.1$; PV-Pemphigus vulgaris; PF-Pemphigus foliaceus

significant higher Dsg3 levels than those with mild or no mucosal involvement $(p<0.01)$. However, Dsg1 ELISA levels did not always correlate with the severity of mucosal involvement $(p>0.1)$ [Table 2].

Analysis showed a direct relationship between the severity of skin involvement and the levels of the Dsg1 antibodies, i.e., patients with moderate and severe disease activity had significant higher levels of Dsg 1 antibodies when compared to those with minimal disease activity $(p<0.01)$. However, ELISA levels of antibodies to Dsg3 did not show a consistent relation to the extent of skin involvement $(p>0.1)$, although patients with extensive disease had slightly more elevated levels of antibodies to Dsg3 [Table 3].

After analyzing the overall relation between the desmoglein antibody levels and disease severity in terms of skin or mucosal involvement, the data was also analyzed to determine whether the relationship between the levels of desmoglein antibodies and disease severity was dependent on the type of pemphigus. The difference between PF and PV was not significant when the severity of skin involvement and Dsg-1 antibody levels were correlated $(p>0.1)$.

However, the presence of severe mucosal involvement correlated well with increased Dsg3 antibody values in PV patients $(p<0.05)$. All six of our patients with PF (none with mucosal involvement) had detectable levels of Dsg3 antibodies.

\section{DISCUSSION}

The autoimmune target of pemphigus is desmoglein, a cadherin type of cell-to-cell adhesion molecule found in the desmosomes. There are three isotypes of desmoglein: Dsg1, Dsg2 and Dsg3. Dsg1 and Dsg3 are usually restricted to the stratified squamous epithelia, where blister formation is located in pemphigus. ${ }^{[7]}$ Patients with pemphigus have anti-Dsg 1 and/or anti-Dsg3 $\lg G$ autoantibodies. $\lg G 1$ and $\lg G 4$ are the most common subclasses of antibodies in patients with active disease, but $\operatorname{IgG} 4$ is more pathogenic and indicates the activity of the disease. Even though PV and PF are distinct diseases, there are several case reports that suggest that a shift between PF and PV may occur. In some of these reports, immunoblotting studies suggested that the antigen has changed in accordance with the change in clinical picture.

Our data demonstrates that in pemphigus, skin disease severity relates to Dsg 1 levels, irrespective of the type of pemphigus (PF/PV). Moreover, Dsg3 levels correlate with mucosal severity in patients with PV. Our findings are in consonance with the study by Harman et al., ${ }^{|6|}$ who also found that levels of antibodies to Dsg 1 correlated with an increase in the severity of skin involvement and elevated anti-Dsg3 antibodies to the severity of mucosal involvement.

Recently, according to the desmoglein compensation theory, pemphigus has been divided into mucosal predominant $\mathrm{PV}$, in which autoantibodies are present only to Dsg3; mucocutaneous PV, where antibodies to both Dsg1 and Dsg3 are present; and PF, where only antibodies to Dsg1 are present and not to Dsg3. ${ }^{[8-10]}$

Correlation of the severity of disease with the level of antibodies detected in patients with PV resulted in two interesting observations: Nine out of the 12 patients with PV having quiescent and resolving disease still had high ELISA values for Dsg3 antibodies, although they had been free from active disease for approximately 6 months or more. The 
reasons for this could be, firstly, the polyclonal nature of the pemphigus autoantibody; therefore, the $\operatorname{Ig} G$ subclass may be an important determinant. Moreover, up to $70 \%$ of the first degree relatives of patients with pemphigus have detectable antibodies that are not of the IgG4 subtype. ${ }^{[11]}$ Secondly, as stated by Harman et al., ${ }^{[6]}$ treatment may have dampened pathogenic mechanisms triggered by antibody binding. Another interesting observation was that all our 6 PF patients, both with active and quiescent disease, demonstrated the presence of autoantibodies to Dsg3. Arteaga et al. demonstrated autoantibodies to Dsg3 in 19 of the 276 patients of PF and fogo selvagem reviewed by them. ${ }^{[12]}$ This suggests that two separate species of antibodies are present rather than one antibody, capable of cross-reacting with Dsg1 and Dsg3. Moreover, their study showed that affinity of purified antibodies to Dsg3 from patients with PF and fogo selvagem by passive transfer induced a PV-like disease in mice. These results suggest that a subset of patients with PF have antibodies to Dsg3 that may also be involved in the pathogenesis of cutaneous disease. Another explanation for this could be that probably there is an epitope shift from PF to PV occurring in these patients. It will be interesting to see if these subsets of patients show a change in their clinical presentation on long-term follow-up (epitope shift phenomenon). Long-term studies are required to confirm these findings.

In a few cases of PV with active disease, low ELISA values for Dsg1 and Dsg3 were found; it is possible that the sera contained pathogenic antibodies to nondesmoglein molecules or to intracellular domain of Dsg1/Dsg3, which could have been undetectable by the ELISA used to detect specific antibodies to only Dsg 1 and Dsg 3.

Our findings suggest that at any given period, the level of the antibodies does correlate with disease severity. However, this may not be true for a small proportion of cases because of the reasons explained above. A detailed prospective study for evaluating the ELISA levels of a given patient for prolonged periods would be helpful in actual correlation with the disease severity and changes that may occur in the spectrum of the disease.

\section{ACKNOWLEDGMENTS}

We are grateful for Dr. Martin Black and Dr. Balbir Bhogal of St John's Institute of Dermatology, St. Thomas Hospital, London, for confirming our ELISA values in their laboratory.

\section{REFERENCES}

1. Chorzelski TP, Von Weiss JF, Lever WF. Clinical significance of autoantibodies in pemphigus. Arch Dermatol 1966;93:570-6.

2. Beutner EH, Jordon RE, Chorzelski TP. The immunopathology of pemphigus and bullous pemphigoid. J Invest Dermatol 1968;51:63-80.

3. Sams WM Jr, Jordon RE. Correlation of pemphigoid and pemphigus antibody titres with activity of disease. BrJ Dermatol 1971;84:7-13.

4. Fitzpatrick RE, Newcomer VD. The correlation of disease activity and antibody titres in pemphigus, Arch Dermatol 1980;116:28590.

5. Ishii K, Amagai M, Hall RP, Hashimoto T, Takayanagi A, Gamou S, et al. Characterization of autoantibodies in pemphigus using antigen-specific enzyme-linked immunosorbent assays with baculovirus-expressed recombinant desmogleins. J Immunol 1997;159:2010-7.

6. Harman KE, Seed PT, Gratian MJ, Bhogal BS, Challacombe SJ, Black MM. The severity of cutaneous and oral pemphigus is related to desmoglein 1 and 3 antibody levels. Br J Dermatol 2001:144:775-80.

7. Amagai M, Klaus-Kovtun V, Stanley JR. Autoantibodies against a novel epithelial cadherin in pemphigus vulgaris, a disease of cell adhesion. Cell 1991;67:869-77.

8. Ding X, Aoki V, Mascaro JM Jr, Lopez-Swiderski A, Diaz LA, Fairley JA. Mucosal and mucocutaneous (generalized) pemphigus vulgaris show distinct autoantibody profiles.J Invest Dermatol 1997;109:592-6.

9. Amagai M, Tsunoda K, Zillikens D, Nagai T, Nishikawa T. The clinical phenotype of pemphigus is defined by the antidesmoglein autoantibody profile. J Am Acad Dermatol 1999;40:167-70.

10. Harman KE, Gratian MJ, Bhogal BS, Challacombe SJ, Black MM. A study of desmoglein 1 autoantibodies in pemphigus vulgaris: racial differences in frequency and the association with a more severe phenotype. Br J Dermatol 2000;143:343-8.

11. Kricheli D, David M, Frusic-Zlotkin M, Goldsmith D, Rabinov $\mathrm{M}$, Sulkes J, et al. The distribution of pemphigus vulgaris-lgG subclasses and their reactivity with desmoglein 3 and 1 in pemphigus patients and their first-degree relatives. $\mathrm{Br} \mathrm{J}$ Dermatol 2000;143:337-42.

12. Arteaga LA, Prisayanh PS, Warren SJ, Liu Z, Diaz LA, Lin MS. Cooperative Group on Fogo Selvagem Research. A subset of pemphigus foliaceus patients exhibits pathogenic autoantibodies against both desmoglein-1 and desmoglein-3.J Invest Dermatol 2002;118:806-11. 